\title{
PRACTICAL STABILITY AND LIMIT CYCLES OF DITHERED RELAY FEEDBACK SYSTEMS
}

\author{
Luigi Iannelli* $^{\sharp}$, Karl Henrik Johansson ${ }^{\natural}$, Ulf Jönsson ${ }^{\S}$, Francesco Vasca ${ }^{\natural}$ \\ \# Dipartimento di Informatica e Sistemistica, Università di Napoli Federico II \\ Via Claudio, 21 - 80125 Napoli, Italy \\ ${ }^{b}$ Department of Signals, Sensors \& Systems, Royal Institute of Technology \\ Osquldasvägen, 10 - 10044 Stockholm, Sweden \\ $\S$ Division of Optimization and Systems Theory, Royal Institute of Technology \\ Lindstedstvägen 25, - 10044 Stockholm, Sweden \\ ${ }^{\natural}$ Dipartimento di Ingegneria, Università del Sannio \\ Corso Garibaldi, 107 - 82100 Benevento, Italy
}

Keywords: Averaging, dither, limit cycles, nonsmooth systems, relay feedback systems.

\begin{abstract}
A new constructive way for choosing dither amplitude and frequency in order to effectively stabilize relay feedback systems by triangular dither is proposed. It is argued that the proposed averaging approach is not easily extended to square dither signals. Trajectories composed by smooth and switching parts are also analyzed and their relationship with limit cycles exhibited by the smoothed system is investigated.
\end{abstract}

\section{Introduction}

High-frequency dither signals are commonly used to compensate for nonlinearities in feedback control systems. The idea is that by injecting a suitably chosen high-frequency signal in the control loop, the nonlinear sector is effectively narrowed and the system can thereby be stabilized. A wide range of examples are found in the areas of electronics [1, 2, 3] and mechanics $[4,5]$. Systems with smooth nonlinearity can be analyzed using powerful methods from the literature $[6,7,8]$. The smoothness assumptions are however often violated in practice [9]; for example, by nonsmooth static components such as switches and relays. For classes of nonsmooth systems with high-frequency excitation, rigorous averaging analysis exists [1, 10]. In the past dither tuning for nonsmooth systems have been mainly limited to describing function methods $[11,12]$. In contrast to these approximate schemes, recently, new dither design methods based on averaging analysis and state-space approach were proposed for relay feedback systems with triangular dither [13], leading to design rules that guarantee stability. In this paper, these results are improved by exploiting a stability condition based on a linear matrix inequality (LMI). A new constructive way for choosing dither amplitude and frequency to stabilize relay feedback systems is proposed. The method is considerably less conservative than [13]. It is also argued that the averaging analysis carried

*Corresponding author. Tel.: +39 081 7683843, Fax: +39081 7683816, e-mail: luiannel@unina.it

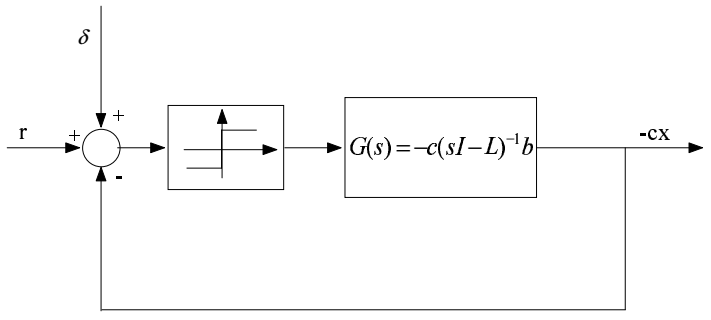

Figure 1: Dithered system.

out for triangular dither is not easily extended to square dither signals. Further it is shown that if the smoothed system has a limit cycle, then for sufficiently small dither period the dithered system will exhibit a smooth-switching trajectory near the state trajectory of the smoothed system. The reason for this is explained and some peculiar behaviors of relay feedback systems with square dither are analyzed. We hence conclude that the behavior of a dithered relay feedback system is highly affected by the shape of the dither signal. This is in stark contrast to systems with Lipschitz continuous dynamics for which it can be shown that the form of the dither signal is not critical at all, see $[6,7]$.

The outline of the paper is as follows. The considered relay feedback system and dither signals are introduced in Section 2, where also averaging analysis is briefly described. Tracking results for relay feedback systems with triangular dither are given in Section 3, together with a method for tuning the parameters of the dither signal. Section 4 analyses stability of limit cycles in dithered systems. The paper is concluded in Section 5, where topics for future work are discussed.

\section{Preliminaries}

The dynamics of the dithered system (see Figure 1) can be described by the differential equation

$$
\dot{x}(t)=L x(t)+b n(c x(t)+r(t)+\delta(t)), \quad x(0)=x_{0} .
$$

Here $L, b$, and $c$ are constant matrices of dimensions $q \times q$, $q \times 1$, and $1 \times q$, respectively. The nonlinearity $n: \mathbb{R} \rightarrow \mathbb{R}$ is 
given by the relay characteristic

$$
n(z)=\operatorname{sgn}(z)= \begin{cases}1, & z>0 \\ 0, & z=0 \\ -1, & z<0\end{cases}
$$

The signal $r(t)$ is the external reference and it is assumed to be Lipschitz continuous, i.e., there exists a constant $M_{r}>0$ such that $\left|r\left(t_{1}\right)-r\left(t_{2}\right)\right| \leq M_{r}\left|t_{1}-t_{2}\right|, \forall t_{1}, t_{2}>0$.

The dither signal $\delta:[0, \infty) \rightarrow \mathbb{R}$ is assumed to be periodic with amplitude $A>0$ and period $p>0$. Usually it is of high frequency compared to the linear dynamics. In the paper we consider triangular and square dither signals. The triangular waveform is defined as

$$
\delta(t)= \begin{cases}\frac{4 A}{p} t, & t \in[0, p / 4) \\ -\frac{4 A}{p} t+2 A, & t \in[p / 4,3 p / 4) \\ \frac{4 A}{p} t-4 A, & t \in[3 p / 4, p)\end{cases}
$$

and the square wave as

$$
\delta(t)= \begin{cases}A, & t \in[0, p / 2) \\ -A & t \in[p / 2, p)\end{cases}
$$

The dithered relay feedback system is assumed to have a solution $x:[0, \infty) \rightarrow \mathbb{R}^{n}$ (in a classical sense), which on every compact subinterval of $[0, \infty)$ is $C^{1}$ everywhere except at finitely many points.

The smoothed system is defined as

$$
\dot{w}(t)=L w(t)+b N(c w(t)+r(t)), \quad w(0)=w_{0},
$$

where the smoothed nonlinearity $N: \mathbb{R} \rightarrow \mathbb{R}$ is the average $N(z)=p^{-1} \int_{0}^{p} n(z+\delta(t)) d t$. For the triangular dither, it is easy to show that

$$
\begin{aligned}
N(z) & =p^{-1} \int_{0}^{p} \operatorname{sgn}(z+\delta(t)) d t=\operatorname{sat}(z / A) \\
& = \begin{cases}1, & z>A \\
z / A, & |z| \leq A \\
-1, & z<-A,\end{cases}
\end{aligned}
$$

and similarly for square dither,

$$
N(z)= \begin{cases}1, & z>A \\ 1 / 2, & z=A \\ 0, & |z|<A \\ -1 / 2, & z=-A \\ -1, & z<-A\end{cases}
$$

Note that for triangular dither $N$ is continuous, while for square dither $N$ is discontinuous.

\section{Averaging Analysis}

In this section we consider the case when the dither is the triangular signal in (2). Under appropriate regularity conditions, the smoothed system approximates the dithered system to any degree of accuracy if the dither period is chosen sufficiently small, as was shown in [13]. That result however provides a conservative bound on the dither period $p$, since no particular attention was paid to the closed-loop dynamics in its proof. Here we will improve the bound on $p$ considerably in the case when the smoothed system is incrementally exponentially stable. This result is important in the design of the dither signal.

\subsection{Tracking}

Over finite time horizon one can prove the following

Theorem 3.1 Let $T, \varepsilon>0$ and $x_{0} \in \mathbb{R}^{n}$ be given. Suppose $r(t)$ is globally Lipschitz with Lipschitz constant $M_{r}$. Moreover, suppose there exist $P=P^{T}>0$ and $\gamma>0$ such that

$$
\left[\begin{array}{ccc}
L^{T} P+P L & P b+c^{T} & 0 \\
b^{T} P+c & -2 A & c \\
0 & c^{T} & -\gamma I
\end{array}\right] \leq 0 .
$$

Then for all $p \in\left(0, p_{0}\right)$,

$$
\left|x\left(t, x_{0}\right)-w\left(t, x_{0}\right)\right| \leq \varepsilon, \quad \forall t \in[0, T]
$$

where

$$
p_{0}=\min \left(\frac{4 A}{7 M}, \Gamma^{-1} \varepsilon\right)
$$

with

$$
\Gamma=c_{3} T+2 c_{1}+\sqrt{\frac{\gamma}{3 c_{3} \lambda_{\min }(P)}\left(\left(c_{3} T+2 c_{1}\right)^{3}-8 c_{1}^{3}\right)}
$$

and $c_{1}, c_{2}$ and $c_{3}$ are constants depending on the system matrices and $M$ is a constant related to the system and to the reference signal $r(t)$.

Proof: See [14].

The next result shows that the state of the smoothed system can track the state of the dithered system arbitrarily well over an infinite time horizon, provided that the dither signal is chosen appropriately. Let us denote by $x\left(t, r, x_{0}\right)$ the solution of (1) with initial condition $x(0)=x_{0}$ and reference $r$. The solution of (4) is similarly denoted $w\left(t, r, w_{0}\right)$. We want to evaluate the approximation error $\left|x\left(t, r, x_{0}\right)-w\left(t, r, x_{0}\right)\right|, \forall t \geq 0$, for any Lipschitz continuous reference signal $r(t)$. The dither amplitude $A$ is tuned such that the smoothed system is incrementally exponentially stable.

Definition 3.1 The system (4) is incrementally exponentially stable if there exist a decay rate $\alpha>0$ and a gain $\beta \geq 1$, such that for any given initial conditions $w_{1}$ and $w_{2}$, the corresponding solutions satisfy

$$
\left|w\left(t, r, w_{1}\right)-w\left(t, r, w_{2}\right)\right| \leq \beta e^{-\alpha t}\left|w_{1}-w_{2}\right| \quad \forall t \geq 0
$$


A simple and often very useful sufficient condition for incremental exponential stability is given by the next lemma.

Lemma 3.1 Assume there exist $Q>0$ and $\alpha>0$ such that the matrix inequality

$$
\left[\begin{array}{cc}
L^{T} Q+Q L+2 \alpha Q & Q b+c^{T} \\
b^{T} Q+c & -2 A
\end{array}\right] \leq 0
$$

holds. Then the smoothed system (4) is incrementally exponentially stable with decay rate $\alpha$ and gain $\beta=$ $\sqrt{\lambda_{\max }(Q) / \lambda_{\min }(Q)}$.

Next we assume that an estimate of the norm $w_{\infty}=$ $\sup _{t \in[0, \infty)}|w(t)|$ is available. (Such a bound can easily be obtained for a given reference signal.)

Theorem 3.2 Let $\varepsilon>0$ and $x_{0} \in \mathbb{R}^{n}$ be given. Suppose that the smoothed system (4) is incrementally exponentially stable with decay rate $\alpha$ and gain $\beta$. Suppose further that $r(t)$ is globally Lipschitz with Lipschitz constant $M_{r}$ and that the bound on the smoothed system state is $w_{\infty}$. Then there exists a $p_{0}$ such that for all $p \in\left(0, p_{0}\right)$,

$$
\left|x\left(t, r, x_{0}\right)-w\left(t, r, x_{0}\right)\right| \leq \varepsilon, \quad \forall t \in[0, \infty) .
$$

Proof: See [14].

The Theorem 3.2 is not only an extension of Theorem 3.1 over the infinite time horizon, since it gives a bound on $p_{0}$ that in general is less conservative than Theorem 3.1 (see [14]).

\subsection{Design of Triangular Dither}

The dither design is a compromise between the conflicting consequences on the control performance of the dither amplitude $A$ and period $p$. A tuning algorithm is obtained by first choosing $A$ such that the smoothed system is exponentially stable, and then $p$ based on the obtained exponential stability parameters $\alpha$ and $\beta$ together with the previous results. For given tracking accuracy $\varepsilon$, Theorem 3.2 gives an upper bound $p_{0}$ for $p$. We would like to optimize the free parameters $\alpha$ and $\mu$ since they are related to $p_{0}$ (see [14], [15] for an explicit relation), such that $p_{0}$ becomes as large as possible. This is hard since the dependence on the free parameters is nonconvex. One way to obtain a reasonable solution is to first pick some $\mu \in(0,1)$. Then choose a dither amplitude $A$ and desired exponential decay rate $\alpha$ such that the LMIs (5) and (7) are feasible. From Theorem 3.1 and 3.2 one can show that $\beta$ and $\gamma / \lambda_{\min }(P)$ should be as small as possible (see [14], [15]). This can be done by solving the following two optimization problems

$$
\begin{aligned}
& \min \beta^{2} \\
& \text { s.t. } \\
& \qquad\left[\begin{array}{cc}
L^{T} Q+Q L+2 \alpha Q & Q b+c^{T} \\
b^{T} Q+c & -2 A
\end{array}\right] \leq 0 \\
& Q \geq \lambda_{\min } I, \quad \lambda_{\min } \beta^{2} I \geq Q,
\end{aligned}
$$

$$
\begin{aligned}
& \min \bar{\lambda} \\
& \text { s.t. } \\
& {\left[\begin{array}{ccc}
L^{T} P+P L & P b+c^{T} & 0 \\
b^{T} P+c & -2 A & c \\
0 & c^{T} & -\gamma I
\end{array}\right] \leq 0} \\
& \quad \bar{\lambda} P \geq \gamma I, \quad \gamma>0
\end{aligned}
$$

by bisection on $\beta^{2}$ and on $\bar{\lambda}$. From the last optimization problem we obtain $\gamma / \lambda_{\min }(P) \leq \bar{\lambda}$. Note that the constraints of these two optimization problems are LMIs for fixed $\beta^{2}$ and $\bar{\lambda}$, respectively. We have arrived at the following tuning algorithm for the triangular dither amplitude and period:

Step 1 Choose $\mu \in(0,1)$.

Step 2 Choose a desired exponential decay rate $\alpha$ and then select the dither amplitude $A$ so that the LMIs (8b) and (9b) are feasible.

Step 3 Solve the optimization problem (8a), which gives $Q$ and $\beta$ and then problem (9a), which gives $P$ and $\gamma / \lambda_{\min }(P)$.

Step 4 Compute $p_{0}$ (the explicit expression of $p_{0}$ is reported in [14]).

For the case when $G(s)=\frac{s-1}{(s+1)^{2}}$ in Figure 1 we get a four order of magnitude better estimate of the dither period compared to the estimate that can be derived from [13]. The bound depends critically on the estimate of $w_{\infty}$ used in Theorem 3.2 and for the initial condition we considered it gives a bound on $p_{0}$ that is almost three orders of magnitude conservative compared to what more heuristic estimates gives.

\section{Limit Cycle Behaviour}

\subsection{Square Wave Dither Signal}

As a motivating example let us now consider the case in which the dither signal is a $p$-periodic square wave (3). Consider an example with

$$
L=\left[\begin{array}{cc}
-2 & -1 \\
1 & 0
\end{array}\right], \quad b=\left[\begin{array}{l}
1 \\
0
\end{array}\right], \quad c=\left[\begin{array}{ll}
1 & -1
\end{array}\right] .
$$

Figure 2 shows the output of the relay feedback system dithered with a square wave and the output of the corresponding smoothed system. The smoothed system is stable while the dithered relay feedback system exhibits what we will call in the sequel a smooth-switching trajectory. In particular, a zoom on Figure 2 provides the plot reported in Figure 3 which shows that the trajectory exhibited by the dithered relay feedback system can be interpreted as a sequence of time intervals. In each of these time intervals the trajectory can be divided into a first part characterized by no switchings (we will call this the smooth part of the trajectory) and a second part where several switchings occur (the switching part of the trajectory). By decreasing the dither period the shape of the waveforms does not 


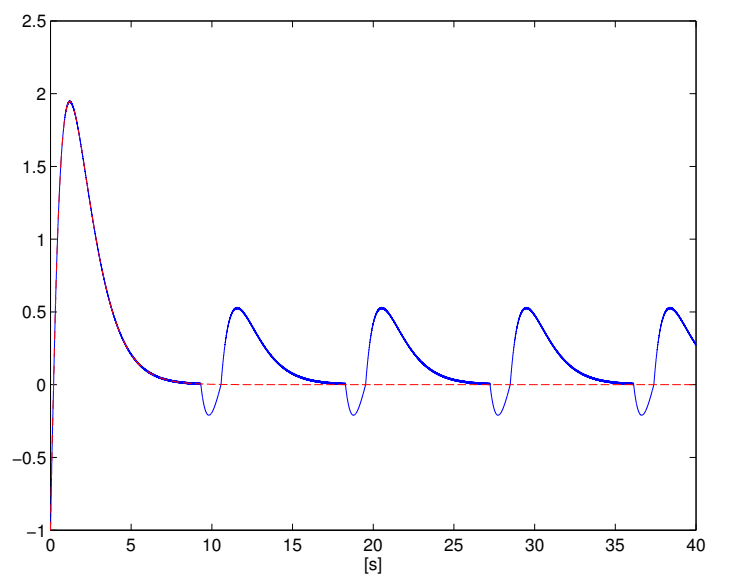

Figure 2: Outputs of the square wave dithered relay feedback system (solid) and the smoothed system (dashed) with dither period $p=1 / 50$, dither amplitude $A=1$ and external reference $r(t)=1$.

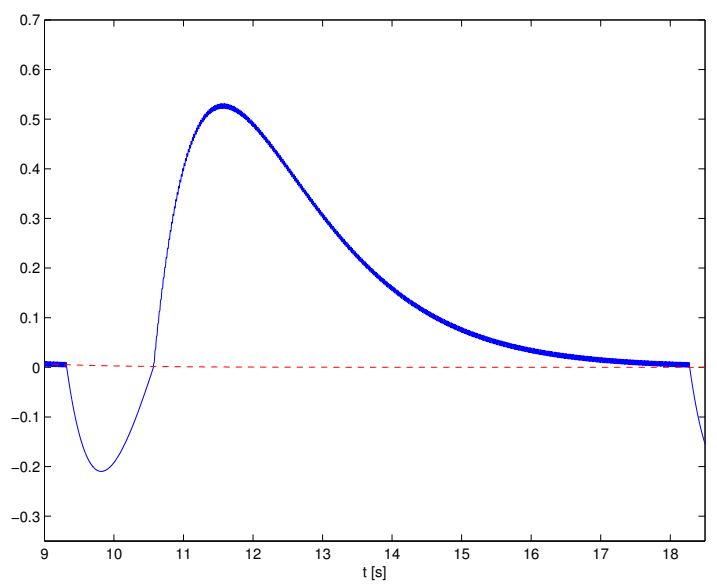

Figure 3: Zoom of Figure 2.

change. The example hence shows that the square dither does not give a similar result as in the triangular dither case: the error between the dithered and smoothed system is not of order $p$ for the square wave dithered system. This is not a coincidence, as is further discussed in [14]. It is interesting to investigate in more details the so-called smooth-switching trajectories. Let us consider a constant reference signal $r(t)=R$ and define hyperplanes $S_{1}=\{x: c x+R+A=0\}$ and $S_{2}=\{x: c x+R-A=0\}$, regions $\Omega_{0}, \Omega_{1}$ and $\Omega_{2}$ and equilibrium points $P_{0}, P_{1}$ and $P_{2}$ as follows (see Figure 4):

- Region $\Omega_{1}=\{x: c x+R+A<0\}$. In this region $u=$ -1 and the dithered system coincides with the smoothed system: $\dot{x}=L x-b$. The equilibrium point is $P_{1}: \bar{x}=L^{-1} b$ (by assuming $L$ invertible).

- Region $\Omega_{2}=\{x: c x+R-A>0\}$. In this region $u=$ +1 and the dithered system coincides with the smoothed system: $\dot{x}=L x+b$. The equilibrium point is $P_{2}: \bar{x}=$ $-L^{-1} b$.

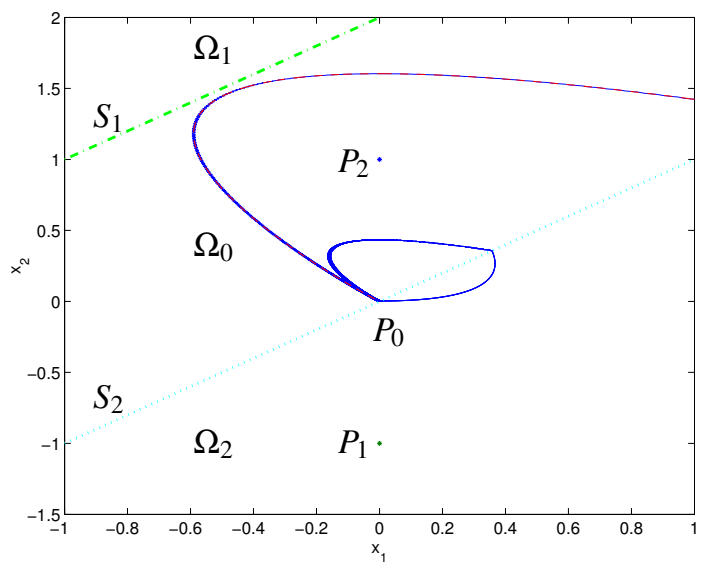

Figure 4: Phase plane portrait of the simulation of Figure 2. The dash-dotted line is the switching plane $c x+A+R=0$ and the dotted line is the switching plane $c x-A+R=0$. In the diagram the equilibrium points $\bar{x}= \pm L^{-1} b$ are also plotted.

- Region $\Omega_{0}=\{x:|c x+R|<A\}$. In this region the dithered system has an input that switches periodically between -1 and +1 (note that while $x \in \Omega_{0}$ the switches are periodic because they only depend on the dither signal and are independent from the state $x$ ). The smoothed system has an input equal to zero: $\dot{w}=L w$. The equilibrium point of the smoothed system is the origin $P_{0}$.

Figure 4 shows the phase portrait corresponding to the simulation in Figure 2. We see that in order to avoid the convergence of the trajectory towards an equilibrium point, it is necessary that $P_{1} \notin \Omega_{1}$ and $P_{2} \notin \Omega_{2}$. These conditions can be satisfied by suitably choosing the parameters $A$ and $R$.

\subsection{Triangular Dither Signal}

In the previous example we have shown that in the case of a square wave dither if $P_{0} \in S_{2}$ it is possible to have smoothswitching trajectories also with high dither frequency. Instead, in order to have smooth-switching trajectory with triangular dither it is not sufficient to put the equilibrium point $P_{0}$ (equilibrium point of the smoothed system in the region $\Omega_{0}$ ) close to $S_{2}$ (or $S_{1}$ ). In fact, in this case the smoothed system (saturation as equivalent nonlinearity) has a continuous vector field on $S_{2}$. The continuity of the vector field does not allow to have a cyclic behavior of the dithered system because the equilibrium point $P_{2} \rightarrow P_{0}$ when $P_{0}$ tends towards $S_{2}$. In fact $P_{0}$ is $\bar{w}=-(L+b c / A)^{-1} b R / A$, a continuous (and linear) function of $R$ and $P_{2}$ is $-L^{-1} b$. It can be shown (by applying the matrix inversion lemma) that when $P_{0} \in S_{2}$ (i.e. $c \bar{w}+R-A=0$ ), $P_{0} \equiv P_{2}$. In the case of the square dither, indeed, both $P_{0}$ and $P_{2}$ didn't depend on $R$ and they were always different points. Inspired by the averaging theorem we can conjecture that a smooth-switching trajectory in a relay feedback system with triangular dither may exist if the smoothed system has a limit cycle that crosses the switching line $S_{2}$. This intuition derives from the averaging theorem although the theorem has not been 


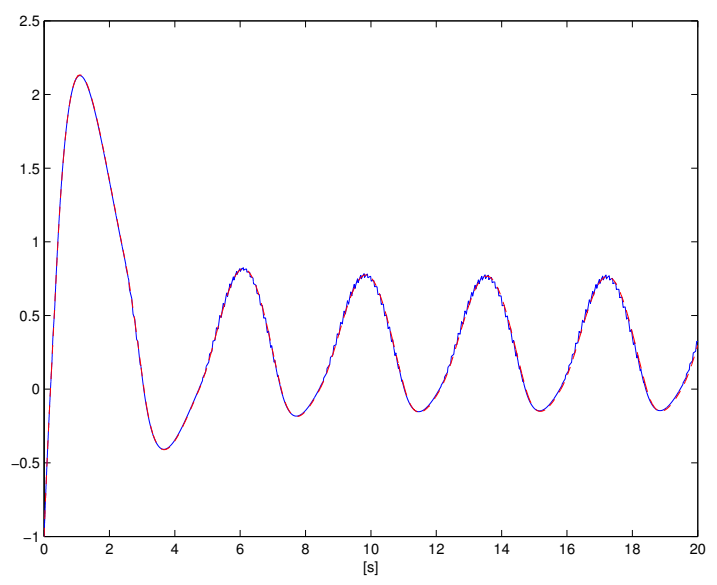

Figure 5: Outputs of the triangular dithered relay feedback system (solid) and the smoothed system (dashed) with dither period $p=1 / 10$, dither amplitude $A=0.45$ and external reference $r(t)=0.45$.

proved (for infinite time horizon) in the case of an unstable smoothed system (the case in which it presents a limit cycle). Figure 5 confirms our intuition.

\subsection{Stability of Limit Cycles}

Assume the smoothed system has a $T^{0}$ periodic limit cycle $w^{0}$ with corresponding closed orbit $\gamma=\left\{w^{0}(t)\right\}$. We say that $w^{0}$ is an exponentially stable limit cycle if there exist $\alpha_{0}>0$ and $\beta_{0} \geq 1$ such that

$$
d(w(t), \gamma) \leq \beta_{0} e^{-\alpha_{0} t} d\left(w_{0}, \gamma\right), \quad \forall t \geq 0
$$

where $d(w, \gamma)=\inf _{y \in \gamma}|w-y|$.

It is in general difficult to check exponential stability of a limit cycle in this global sense. The result below can be modified to the case of locally exponentially stable limit cycle but for simplicity we consider the global assumption.

Proposition 4.1 Suppose $r(t)=R$ and that the smoothed system (4) has an exponentially stable limit cycle $w^{0}$ with orbit $\gamma$. Then there exists $p_{0}$ such that for $p \in\left(0, p_{0}\right)$, there exist $\alpha>0$ and $\beta \geq 1$

$$
d(x(t), \gamma) \leq \beta e^{-\alpha t} d\left(x_{0}, \gamma\right)+\varepsilon
$$

Proof: See the appendix.

The simulations in Figure 6 show the convergence of the smoothed system to a limit cycle and of the dithered system to a smooth-switching orbit for the case when $w_{0} \neq x_{0}$. In Figure 7 we can see what happens in the time domain.

\section{Conclusions}

Dither is often used in practice in order to stabilize nonsmooth systems. In this paper a procedure for the design of the amplitude and the period of a triangular dither signal to stabilize

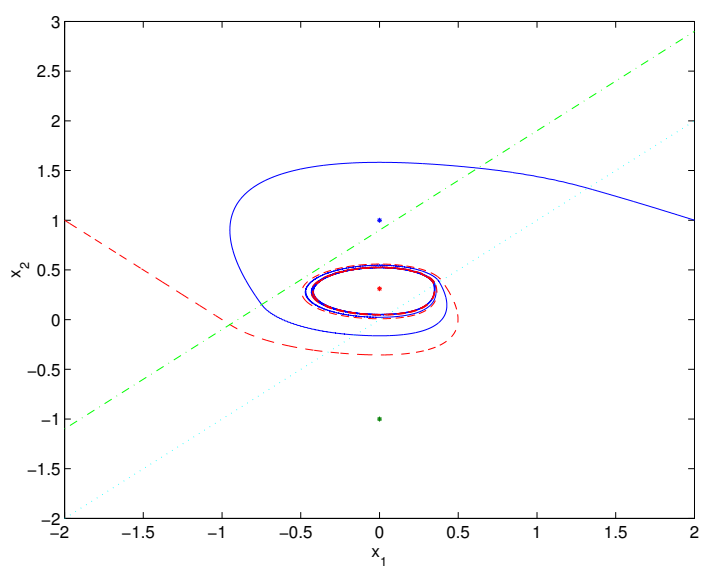

Figure 6: Phase portrait of the simulation corresponding to Figure 5 for different initial conditions.

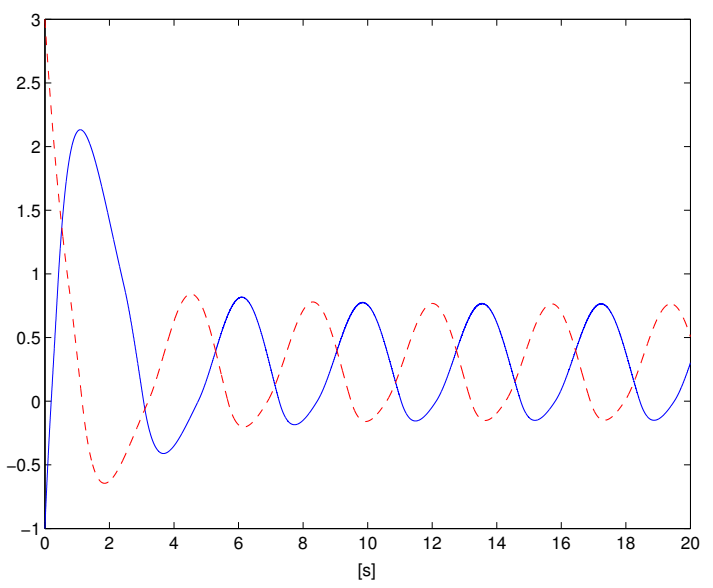

Figure 7: Simulation corresponding to Figure 5 for different initial conditions.

relay feedback systems has been proposed. The tuning algorithm is based on an averaging approach and exploits LMIs. The averaging approach can also be useful when the emphasis is on more complex nonlinear phenomena exhibited by the relay feedback system. In particular, we have shown that in the presence of triangular dither, the existence of an exponentially stable limit cycle in the smoothed system predicts the existence of smooth-switching trajectories in the dithered system. Some examples have highlighted that the averaging approach cannot be simply extended to the case of square wave and more general zero-slope dither signals. Further investigations in this direction are the main object of some ongoing works $[16,17]$ in which we deal with a wider class of switched systems and with more general dither signals.

\section{Acknowledgements}

The work by L. Iannelli and F. Vasca was partially supported by the European Commission within the SICONOS project. The work by K. H. Johansson and U. Jönsson was supported by the European Commission within the RECSYS project and the 
Swedish Research Council.

\section{Appendix: Proof of Proposition 4.1}

By hypothesis the system (4) with $r(t)=R$ has a limit cycle $\gamma$ that is exponentially stable. Hence, there exist $\alpha_{0}>0$ and $\beta_{0} \geq 1$ such that (11) holds.

We iteratively consider time intervals of length $T$ and, in order to guarantee a decay rate of 0.1 , we choose $T=$ $-\alpha_{0}{ }^{-1} \ln \left(0.1 / \beta_{0}\right)$. Then, (from Theorem 1 in [13]) if $p_{0}$ is sufficiently small, we have

$$
|x(t)-w(t)| \leq \varepsilon_{0}
$$

on $t \in[0, T]$. If we consider a new smoothed system satisfying (4) on the time interval $[k T,(k+1) T], k=0,1,2, \ldots$, with initial condition $w(k T)=x(k T)$, then it follows from the above arguments that

$$
d(w(t), \gamma) \leq \beta_{0} e^{-\alpha_{0}(t-k T)} d(x(k T), \gamma), \quad \forall t \geq k T,
$$

and, by applying the averaging theorem on finite time horizon again,

$$
\begin{aligned}
d(x(t), \gamma) & \leq|x(t)-w(t)|+d(w(t), \gamma) \leq \varepsilon_{0}+d(w(t), \gamma) \\
& \leq \beta_{0} e^{-\alpha_{0}(t-k T)} d(x(k T), \gamma)+\varepsilon_{0}
\end{aligned}
$$

on $t \in[k T,(k+1) T]$. By evaluating (12) in $t=(k+1) T$,

$$
d(x((k+1) T), \gamma) \leq 0.1 d(x(k T), \gamma)+\varepsilon_{0} .
$$

Hence

$$
d(x(k T), \gamma) \leq 0.1^{k} d\left(x_{0}, \gamma\right)+\varepsilon_{0} \frac{1-0.1^{k}}{1-0.1} .
$$

Then (12) becomes

$$
\begin{aligned}
d(x(t), \gamma) & \leq \beta_{0} e^{-\alpha_{0}(t-k T)}\left(e^{-\alpha k T} d\left(x_{0}, \gamma\right)+\frac{\varepsilon_{0}}{0.9}\right)+\varepsilon_{0} \\
& \leq \beta_{0} e^{-\alpha_{0}(t-k T)} e^{-\alpha k T} d\left(x_{0}, \gamma\right)+\underbrace{\beta_{0} \frac{\varepsilon_{0}}{0.9}+\varepsilon_{0}}_{\varepsilon},
\end{aligned}
$$

where $\alpha=-T^{-1} \ln 0.1$. Since $\alpha_{0}>\alpha$ and $t \geq k T$, (15) becomes

$$
d(x(t), \gamma) \leq \beta_{0} e^{-\alpha t} d\left(x_{0}, \gamma\right)+\varepsilon .
$$

We have thus shown practical stability with $\alpha=-T^{-1} \ln 0.1$ and $\beta=\beta_{0}$.

\section{References}

[1] B. Lehman and R. Bass, "Extensions of averaging theory for power electronics systems", IEEE Transactions on Power Electronics, vol. 11, no. 4, pp. 542-553, 1996.

[2] A. V. Peterchev and S. R. Sanders, "Quantization resolution and limit cycling in digitally controlled PWM converters", in Proc. IEEE Power Electronics Specialists Conf., Vancouver, Canada, June 2001.
[3] R. M. Gray and D. L. Neuhoff, "Quantization", IEEE Transactions on Information Theory, vol. 44, no. 6, pp. 2325-2383, 1998.

[4] B. Armstrong-Helouvry, Control of Machines with Friction, Kluwer Academic Publisher, Boston, 1991.

[5] B. Armstrong-Helouvry, P. Dupont, and C. Canudas de Wit, "A survey of models, analysis tools and compensation methods for control of machines with friction", Automatica, vol. 30, no. 7, pp. 1083-1138, 1994.

[6] G. Zames and N. A. Shneydor, "Dither in non-linear systems", IEEE Transactions on Automatic Control, vol. 21, no. 5, pp. 660-667, October 1976.

[7] G. Zames and N. A. Shneydor, "Structural stabilization and quenching by dither in non-linear systems", IEEE Transactions on Automatic Control, vol. 22, no. 3, pp. 352-361, June 1977.

[8] S. Mossaheb, "Application of a method of averaging to the study of dither in non-linear systems", International Journal of Control, vol. 38, no. 3, pp. 557-576, September 1983 .

[9] A. A. Pervozvanski and C. Canudas de Wit, "Asymptotic analysis of the dither effect in systems with friction", $A u$ tomatica, vol. 38, no. 1, pp. 105-113, January 2002.

[10] A. Kh. Gelig and A. Churilov, Stability and Oscillations of Nonlinear Pulse Modulated Systems, Birkhäuser, Berlin, 1998.

[11] D. Atherton, Nonlinear Control Engineering, Van Nostrand Reinhold Co., London, 1975.

[12] A. Gelb and W. Vander-Velde, Multiple Input Describing Functions, McGraw-Hill, 1968.

[13] L. Iannelli, K.H. Johansson, U. Jönsson, and F. Vasca, "Analysis of dither in relay feedback systems", in Proc. of IEEE Conference on Decision and Control, Las Vegas, Nevada, USA, dec 2002.

[14] L. Iannelli, K. H. Johansson, U. Jönsson, and F. Vasca, "Dither for smoothing relay feedback systems: an averaging approach", IEEE Transactions on Circuits and Systems, Part I, 2003, to appear.

[15] L. Iannelli, Dither for Smoothing Relay Feedback Systems: an Averaging Approach, $\mathrm{PhD}$ thesis, University of Napoli "Federico II", Napoli, Italy, Nov. 2002, http://cds.unina.it/ luiannel/eng/public.htm.

[16] L. Iannelli, K. H. Johansson, U. Jönsson, and F. Vasca, "Effects of dither shapes in nonsmooth feedback systems: experimental results and theoretical insight", submitted to CDC03, 2003.

[17] L. Iannelli, K. H. Johansson, U. Jönsson, and F. Vasca, "Shaping dither signals for switched systems", in preparation. 